\title{
Dickkopf 2 promotes proliferation and invasion via Wnt signaling in prostate cancer
}

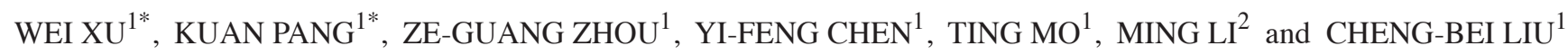 \\ ${ }^{1}$ Department of Urology, The First People's Hospital of Yulin, Yulin, Guangxi 537000; \\ ${ }^{2}$ Basic Medicine College, Shanghai Jiaotong University, Shanghai 200025, P.R. China
}

Received May 13, 2015; Accepted March 1, 2016

DOI: $10.3892 / \mathrm{mmr} .2016 .5502$

\begin{abstract}
Wnt $/ \beta$-catenin signaling dysregulation is involved in tumorigenesis. Furthermore, epigenetic modification of the Dickkopf (DKK) family (DKK1-4) has been shown to be important in the regulation of Wnt signaling. However, the functions and mechanism of DKK2 in the development and progression of prostate cancer remain unclear. Therefore, the present study investigated the role of DKK2 in prostate cancer. The mRNA and protein expression levels of DKK2 in prostate cancer tissues and cells were assessed by reverse transcription-quantitative polymerase chain reaction and western blotting, respectively. The biological function of DKK2 in prostate cancer was investigated using 3-(4,5-dimethylthiazol-2-yl)-2,5-diphenyltetrazolium bromide and transwell invasion assays. DKK2 was demonstrated to be upregulated in prostate cancer tissues and cells, and knockdown of DKK2 suppressed cell proliferation and invasion. Furthermore, small interfering RNA targeting DKK2 inhibited the expression of $\beta$-catenin, cyclin D1 and c-Myc in prostate cancer cells. The present report suggested that DKK2 downregulation suppressed the proliferation and invasion of prostate cancer cells by inhibiting the Wnt/ $\beta$-catenin signaling pathway.
\end{abstract}

\section{Introduction}

Prostate cancer is the most common cancer type diagnosed in men from developed countries and is the leading cause of cancer mortalities worldwide (1). Despite the various treatments for prostate cancer having been improved recently, therapy for patients with advanced prostate cancer still lacks efficacy. This is largely a result of a lack of knowledge

Correspondence to: Dr Cheng-Bei Liu, Department of Urology, The First People's Hospital of Yulin, 495 Middle Jiaoyu Road, Yulin, Guangxi 537000, P.R. China

E-mail: chengbei_liu@163.com

*Contributed equally

Key words: Dickkopf 2, proliferation, Wnt//-catenin signaling, invasion regarding the molecular mechanism underlying the pathogenesis and progression of prostate cancer. Therefore, to improve the understanding of the mechanism associated with prostate cancer progression, it is vital to explore novel therapeutic strategies for patients with prostate cancer.

Accumulating evidence has demonstrated a significant role for the Wnt/ $\beta$-catenin signaling pathway in the development and progression of human cancer (2-4). The Dickkopf (DKK) family is one of the Wnt antagonist families, and inhibits Wnt signaling by binding to the lipoprotein receptor-related protein 5/6 component of the Wnt receptor complex (5). DKK consists of four members, each of which possesses an N-terminal signal peptide and contains two conserved cysteine-rich domains separated by a linker region (6). DKK2 is a secretory protein, and numerous previous studies have shown that it is involved in tumor cell proliferation, survival, migration and invasion. In renal carcinoma, the expression of DKK2 is epigenetically suppressed, and its ectopic expression reduces invasion and induces apoptosis of cancer cells (7). DKK2 also increases tumor growth and metastasis through the transcriptional upregulation of matrix metalloprotease-1 in Ewing's Sarcoma (8). Additionally, high expression of DKK2 has also been reported in colorectal cancer (9). These findings suggested that DKK2 performs an oncogenic or a tumor-suppressing function depending on the cell type or context. However, the functions and mechanism of DKK2 in the development and progression of prostate cancer remain to be elucidated.

The present study found that DKK2 was upregulated in prostate cancer tissues and cells, and small interfering (si) RNA targeting DKK2 inhibited cell growth and invasion. In addition, the underlying molecular mechanism was also assessed in the present study.

\section{Materials and methods}

Human tissue specimens. Human prostate cancer and adjacent non-tumor liver tissue samples were collected from patients undergoing resection of prostate cancer at the Department of Urology, The First People's Hospital of Yulin City (Yulin, China). All tissues were classified according to the World Health Organization criteria and staged according to the tumor-node-metastasis classification. Informed consent was obtained from each patient and the study was approved by the 
Institute Research Ethics Committee of the Department of Urology, The First People's Hospital of Yulin City.

Cell culture. DU-145 and PC-3 human prostate cancer cell lines and the RWPE-1 normal prostate epithelial cell line were purchased from American Type Culture Collection (Manassas, VA, USA) and maintained in Dulbecco's modified Eagle's medium (Invitrogen; Thermo Fisher Scientific, Inc., Waltham, MA, USA), supplemented with $10 \%$ fetal bovine serum (Gibco; Thermo Fisher Scientific, Inc.), $100 \mu \mathrm{g} / \mathrm{ml}$ penicillin and $100 \mu \mathrm{g} / \mathrm{ml}$ streptomycin (Sigma-Aldrich, St. Louis, MO, USA) at $37^{\circ} \mathrm{C}$ in a humidified atmosphere containing $5 \% \mathrm{CO}_{2}$.

Stable transfection with siRNA-DKK2. The prostate cancer cells were plated into 12 -well plates $\left(1 \times 10^{5}\right.$ cells/well) in endothelial cell growth medium-2 (Invitrogen; Thermo Fisher Scientific, Inc.) without antibiotics. After incubation for $24 \mathrm{~h}$, the cells were transfected with siRNA targeting DKK2 (Sangon Biotech Co., Ltd., Shanghai, China) using Lipofectamine 2000 (Invitrogen; Thermo Fisher Scientific, Inc.), according to the manufacturer's protocol.

Reverse transcription-quantitative polymerase chain reaction $(R T-q P C R)$. The total RNA was extracted from primary tumor tissues or cells homogenized in TRIzol reagent (Invitrogen; Thermo Fisher Scientific, Inc.), according to the manufacturer's protocol. Superscript First-Strand kit was used to synthesize first strand cDNA (Invitrogen; Thermo Fisher Scientific, Inc.). RT-qPCR was performed on the Bio-Rad iQ5 Quantitative PCR system (Takara Bio, Inc., Dalian, China). The primers used were as follows: DKK2, sense: 5'-AGTACCCGCTGC AATAATGG-3' and antisense: 5'-GAAATGACGAGCACA GCAAA-3'; $\beta$-actin, sense: 5'-GATCATTGCTCCTCC TGAGC-3' and antisense: 5'-ACTCCTGCTTGCTGATCC AC-3' (Sangon Biotech Co., Ltd.). A total of three independent experiments were performed for each reaction in triplicate.

Western blotting. The total proteins were extracted from cells using radioimmunoprecipitation lysis buffer (Beyotime Institute of Biotechnology, Inc., Nantong, China). Equal quantities of protein sample (30 $\mu \mathrm{g}$ ) were separated on a $10 \%$ sodium dodecyl sulfate-polyacrylamide gel, transferred onto nitrocellulose membranes (EMD Millipore, Boston, MA, USA) and blocked with $10 \%$ non-fat milk in phosphate-buffered saline at $4^{\circ} \mathrm{C}$ overnight. The membranes were subsequently incubated with the following primary antibodies: Monoclonal mouse anti- $\beta$-catenin (1:3,000; sc-53484), monoclonal mouse anti-cyclin D1 (1:3,000; sc-20044), mouse monoclonal anti-c-Myc (1:2,500; sc-47694) and anti- $\beta$-actin (1:1,500; sc-47778; Santa Cruz Biotechnology, Inc., Santa Cruz, CA, USA) overnight at $4^{\circ} \mathrm{C}$. After washing with Tris-buffered saline/ $0.05 \%$ Tween- 20 buffer, the membranes were incubated for $1 \mathrm{~h}$ at room temperature with rabbit anti-mouse $\operatorname{IgG}$ horseradish peroxidase-conjugated secondary antibody (1:3,000; sc-358917; Santa Cruz Biotechnology, Inc.). The membrane was subsequently washed and visualized by enhanced chemiluminescence detection system (GE Healthcare Life Sciences, Little Chalfont, UK).

Cellproliferationassay.The3-(4,5-dimethylthiazol-2-yl)-2,5-diphenyltetrazolium bromide (MTT) assay was used to determine
A

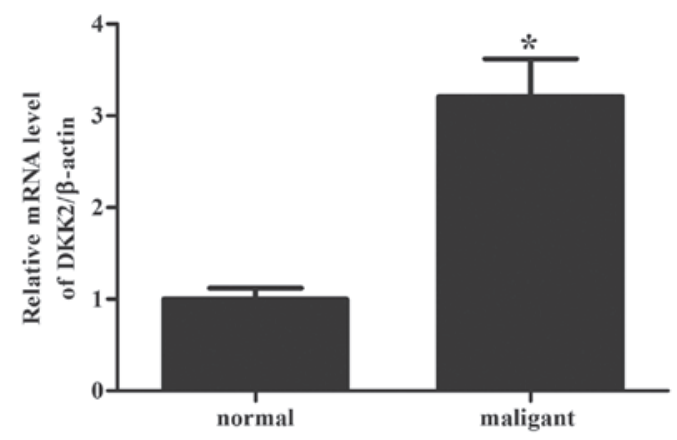

B

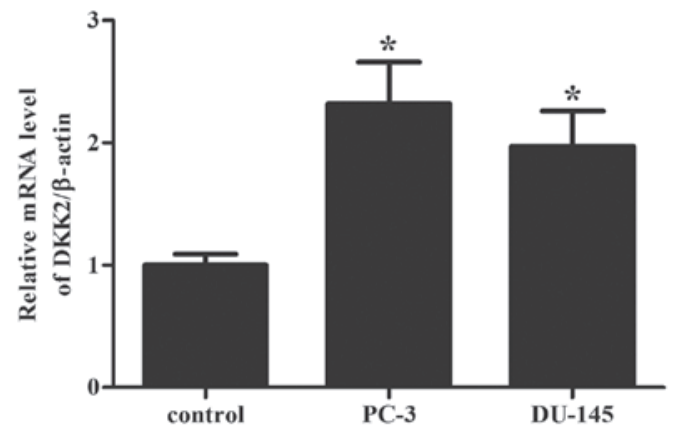

C control PC-3 DU-145

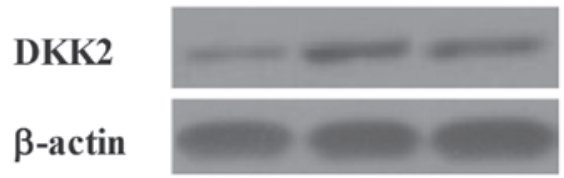

Figure 1. Expression of DKK2 in human prostate cancer tissue samples and cell lines. (A) The mRNA expression of DKK2 was analyzed by reverse transcription-quantitative polymerase chain reaction. The mRNA expression levels of DKK2 in malignant prostate cancer were significantly higher compared with that in normal prostate tissue. (B) The mRNA expression of DKK2 in prostate cancer cell lines. (C) A representative western blotting image of DKK2 protein in prostate cancer cell lines. All experiments were performed in triplicate and the data are shown as the mean \pm standard deviation ( ${ }^{*} \mathrm{P}<0.05$ vs. control). DKK, Dickkopf.

cell proliferation. Briefly, the cells were seeded into 96-well plates at a density of $1 \times 10^{5}$ cells/well. Following treatment with siRNA-DKK2 or siRNA-mock, $20 \mu 1$ MTT $(5 \mathrm{mg} / \mathrm{ml})$ was added to each well and the cells were incubated for a further $6 \mathrm{~h}$ at $37^{\circ} \mathrm{C}$. The supernatant was replaced with $200 \mu 1$ isopropanol to dissolve the formazan product. The cell viability was determined by measuring the absorbance at $490 \mathrm{~nm}$ using an ELISA microplate reader (Abcam, Cambridge, UK). All samples were performed in triplicate and the results are presented as the percentage of growth inhibition.

Cell invasion assay. Cell invasion was measured using a modified Boyden chamber coated with Matrigel (BD Biosciences, Bedford, MA, USA). The cells ( $1 \times 10^{5}$ cells/well) treated with siRNA-DKK 2 or siRNA-mock were seeded into the upper compartment. The medium, including $10 \%$ fetal bovine serum, was added into the lower compartment. After $48 \mathrm{~h}$, the cells that passed through the lower side of the membrane were stained with hematoxylin and eosin (Sigma-Aldrich), and were counted using a microscope. A total of five random fields were selected 
A PC-3
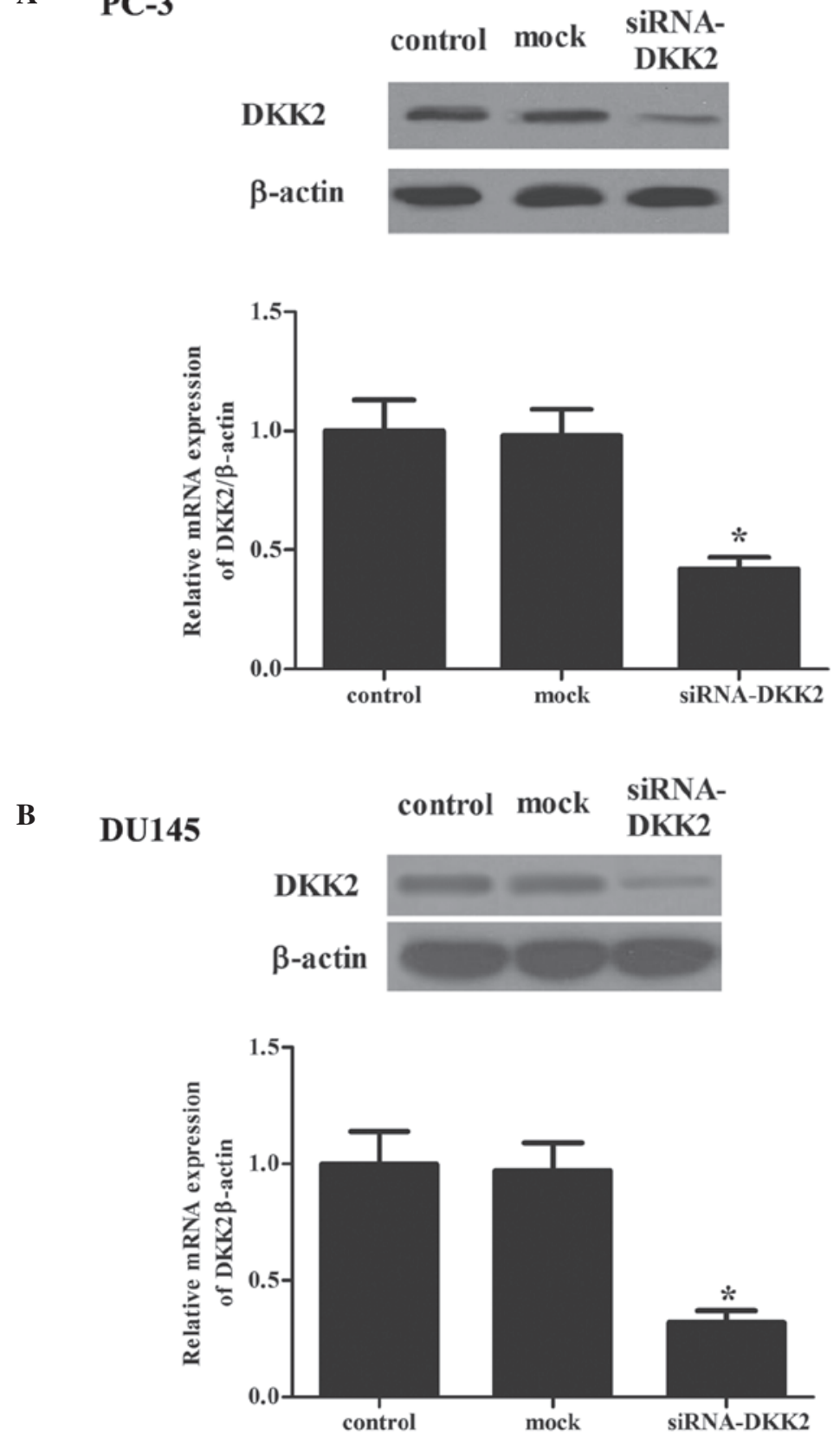

Figure 2. Expression of DKK2 in the PC-3 and DU145 cell lines. The cells were transfected with siRNA-DKK2 and scrambled control DKK2. The corresponding transfection effective was detected by reverse transcription-quantitative polymerase chain reaction and western blotting. The expression was determined in (A) PC-3 and (B) DU145 cells. All experiments were performed in triplicate and the data are shown as the mean \pm standard deviation ("P $<0.05 \mathrm{vs.}$ control and mock). DKK, Dickkopf; si, small interfering.

for each membrane, and the results were expressed as the number of cells migrated per field.

Statistical analysis. All results are presented as the mean \pm standard deviation. Statistical analysis was performed using Student's t test for the comparison of two groups or one-way analysis of variance for multiple comparisons. $\mathrm{P}<0.05$ was considered to indicate a statistically significant difference.

\section{Results}

DKK2 expression levels in prostate cancer tissues and cell lines. To determine the role of DKK2 in prostate cancer, the
mRNA expression levels of DKK2 were compared between prostate cancer tissues and adjacent normal prostate tissues. The mRNA expression of DKK2 was high in prostate cancer tissues compared with that in normal prostate tissues (Fig. 1A). Consistent with the observations from tissue samples, it was demonstrated that the mRNA and protein expression levels of DKK2 were higher in the prostate cancer cells, PC-3 and DU-145, compared with the normal human prostate cells (Fig. 1B and C).

Effects of transfection. It is possible that DKK2 may be important in the development of prostate cancer. Therefore, the present study generated the stable knockdown DKK2 in 

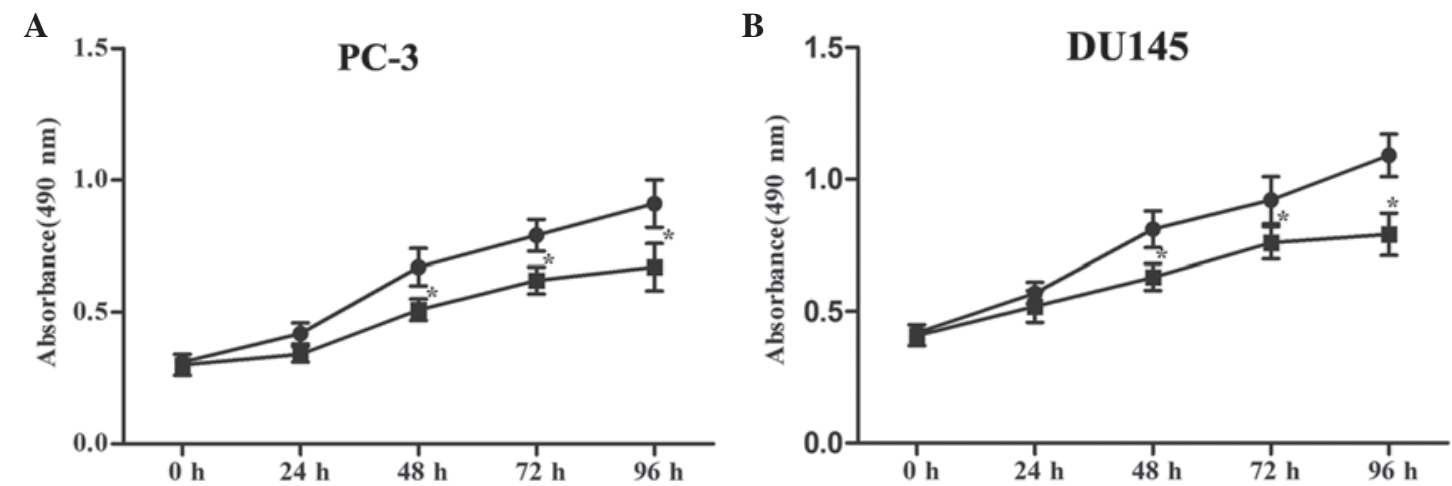

Figure 3. siRNA-DKK2 suppresses the proliferation of prostate cancer cells. The cell proliferation of siRNA-DKK2-transfected cells was markedly reduced in (A) PC-3 and (B) DU145 cells compared with the mock controls. All experiments were performed in triplicate and the data are shown as the mean \pm standard deviation ("P<0.05 vs. mock). DKK, Dickkopf; si, small interfering.

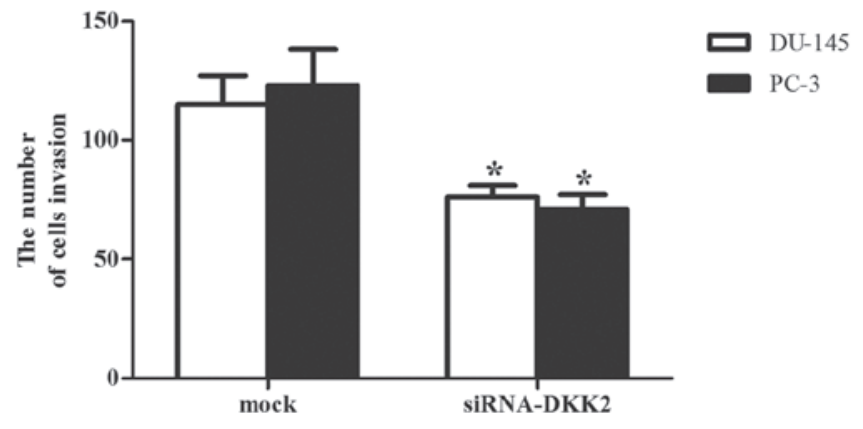

Figure 4. siRNA-DKK2 suppresses the invasion of prostate cancer cells. The cell invasion of siRNA-DKK2-transfected cells was markedly reduced in PC-3 and DU145 cells compared with the mock controls. All experiments were performed in triplicate and the data are shown as the mean \pm standard deviation ("P<0.05 vs. mock). DKK, Dickkopf; si, small interfering.

PC-3 and DU-145 cells. To test the efficiency of DKK2 transfection, RT-qPCR and western blotting were used to determine the mRNA and protein expression levels, respectively. As shown in Fig. 2, the siRNA significantly decreased the protein and mRNA expression levels of DKK2 in PC-3 (Fig. 2A) and DU-145 (Fig. 2B) cells, as compared with the control group.

Effects of DKK2 on the proliferation of prostate cancer cells. To investigate the effects of DKK2 on the proliferation of human prostate cancer cells, the cells were transfected with siRNA-DKK2 or siRNA-mock, and cell proliferation was measured using an MTT assay. As indicated in Fig. 3, compared with the siRNA-mock, siRNA-DKK2 significantly inhibited the proliferation of PC-3 (Fig. 3A) and DU-145 (Fig. 3B) cells, in a time-dependent manner. These results indicated that DKK2 promoted the proliferation of prostate cancer cells.

Effects of DKK2 on the invasion of prostate cancer cells. To assess the effect of DKK2 on prostate cancer cell invasion, the cells were transfected with siRNA-mock or siRNA-DKK2 and were placed into a Boyden chamber coated with Matrigel. As shown in Fig. 4, the number of siRNA-DKK2-transfected PC-3 and DU-145 cells that passed through the Matrigel was significantly inhibited compared with that derived from mock or non-transfected cells $(\mathrm{P}<0.05)$, suggesting that DKK2 enhanced the invasion of prostate cancer cells.
DKK2 exerts its function by promoting $\beta$-catenin signaling. The present study next investigated a potential mechanism for DKK2-mediated cell migration and invasion. It is well known that DKK 2 is a typical inhibitor of Wnt/ $\beta$-catenin signaling pathway, hence the effects of siRNA-DKK2 on the downstream target genes of Wnt pathway, $\beta$-catenin, cyclin D1 and c-Myc, were investigated. As shown in Fig. 5, the expression levels of $\beta$-catenin, cyclin D1 and c-Myc were significantly inhibited by siRNA-DKK2 in PC-3 and DU-145 cells.

\section{Discussion}

The present study found that DKK2 is markedly overexpressed in both prostate cancer tissues and cells. In addition, siRNA-DKK2 significantly suppressed the proliferation and the invasion by inhibiting the $\mathrm{Wnt} / \beta$-catenin signaling pathway.

DKK1 was directly produced by prostate cancer cells, whereas normal prostate tissue did not produce this molecule. DKK1 has been reported to be upregulated in the sera of patients with prostate cancer (10). DKK3 was also upregulated in the reactive stroma of prostate cancer tissue (11). Consistent with the above results, the present study found that DKK2 is overexpressed in both prostate cancer tissues and cells. All findings indicated that DKK2 is an oncogenic factor in prostate cancer.

Cell proliferation and invasion are two of the most important features of malignant cell behavior. DKK3 has been reported to be associated with various cancer types and it is a tumor suppressor for human cancer growth $(12,13)$. However, the function and biological role of DKK2 remains to be elucidated. In Ewing's sarcoma, DKK2 increases the proliferation, anchorage-independent colony formation, osteolysis and invasion of the sarcoma cells. In addition, it regulates the expression levels of different genes important for invasion into bone and osteolysis (8). By contrast, overexpression of DKK2 suppressed malignant cell growth and invasion in human epithelial ovarian cancer cells (14). These findings suggested that DKK2 performs an oncogenic or a tumor-suppressing function dependent on the cell type or context. The present study found that siRNA-DKK2 markedly inhibited the proliferation and invasion of prostate cancer cells, suggesting that DKK2 is important for promoting cell proliferation and invasion of prostate cancer. 
A
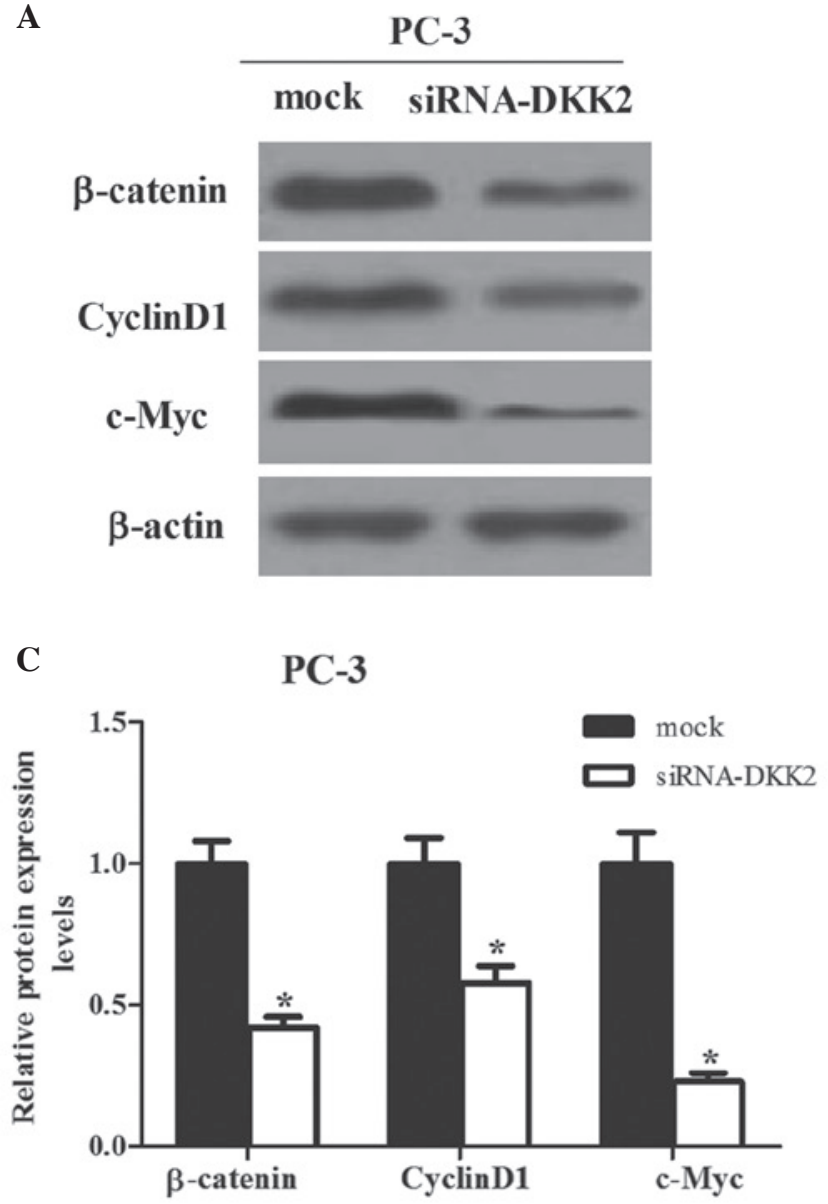

B

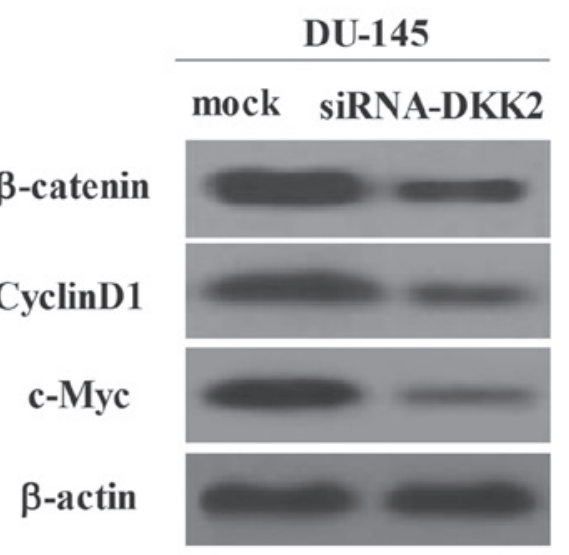

D

DU-145

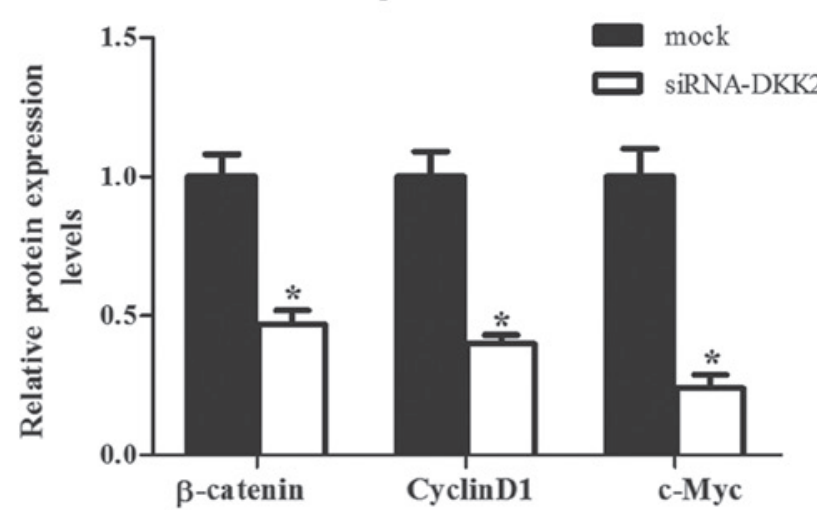

Figure 5. DKK2 exerts its function by promoting the signaling of $\beta$-catenin. Western blotting was performed to assess the expression levels of $\beta$-catenin, cyclin D1 and c-Myc in siRNA-DKK2-transfected (A) PC-3 and (B) DU-145 cells. The relative expression levels of $\beta$-catenin, cyclin D1 and c-Myc was determined in (C) PC-3 and (D) DU-145 cells using Image-Pro Plus 6.0 software. The expression levels were normalized against that of $\beta$-actin All experiments were repeated at least three times and the data are presented as the mean \pm standard error of the mean (" $\mathrm{P}<0.05$ vs. the mock group). DKK, Dickkopf; si, small interfering.

Deregulation of the Wnt/ $\beta$-catenin signaling pathway is a hallmark of various cancer types. $\beta$-catenin is a critical end component of the Wnt signaling pathway, which regulates cell growth, apoptosis and migratory behavior in response to intercellular adhesion molecules (15). It was previously reported that KIF3a, a subunit of kinesin-II motor protein, functions as an agonist of the Wnt signaling pathway, and it increases CK1-dependent DVL2 phosphorylation and $\beta$-catenin activation in prostate cancer cells, leading to transactivation of the Wnt-signaling target genes, including cyclin D1, HEF1 and matrix metalloproteinase 9 (16). Previous studies have shown that the expression levels of Wnt- 1 and $\beta$-catenin were increased in invasive prostate cancer cell lines and in primary prostate cancer specimens (17-20). In line with these previous reports, the present study demonstrated that silencing of DKK2 significantly suppressed the expression of $\beta$-catenin, cyclin D1 and c-Myc in prostate cancer cells. $\beta$-catenin forms a cell adhesion complex with E-cadherin raising the possibility that loss of expression or a change in $\beta$-catenin distribution in the cell can also alter downstream signaling, decreased inter-cellular adhesion and the promotion of metastasis (21). A previous study also demonstrated that a novel small molecule inhibitor of Wnt/ $\beta$-catenin signaling, PKF118-310, inhibited proliferation and Wnt/ $\beta$-catenin signaling in prostate cancer cells (19). These results suggested that the inhibitory effect of siRNA-DKK2 on prostate cancer cell proliferation and invasion may involve the suppression of the $\mathrm{Wnt} / \beta$-catenin signaling pathway.

In conclusion, the present findings demonstrated that DKK2 downregulation suppressed the proliferation and invasion of prostate cancer cells by inhibiting the Wnt/ $\beta$-catenin signaling pathway, which supported the notion that DKK2 may serve as a diagnostic biomarker for monitoring prostate cancer development and progression. Therefore, DKK2 is a potential therapeutic strategy for the treatment of prostate cancer.

\section{References}

1. Jemal A, Bray F, Center MM, Ferlay J, Ward E and Forman D: Global cancer statistics. CA Cancer J Clin 61: 69-90, 2011.

2. Fodde R and Brabletz T: Wnt/beta-catenin signaling in cancer stemness and malignant behavior. Curr Opin Cell Biol 19: 150-158, 2007.

3. Polakis P: Wnt signaling and cancer. Genes Dev 14: 1837-1851, 2000.

4. Maretto S, Cordenonsi M, Dupont S, Braghetta P, Broccoli V, Hassan AB, Volpin D, Bressan GM and Piccolo S: Mapping Wnt/beta-catenin signaling during mouse development and in colorectal tumors. Proc Natl Acad Sci USA 100: 3299-3304, 2003. 
5. Kawano Y and Kypta R: Secreted antagonists of the Wnt signalling pathway. J Cell Sci 116: 2627-2634, 2003.

6. Krupnik VE, Sharp JD, Jiang C, Robison K, Chickering TW, Amaravadi L, Brown DE, Guyot D, Mays G, Leiby K, et al: Functional and structural diversity of the human Dickkopf gene family. Gene 238: 301-313, 1999.

7. Hirata H, Hinoda Y, Nakajima K, Kawamoto K, Kikuno N Kawakami K, Yamamura S, Ueno K, Majid S, Saini S, et al: Wnt antagonist gene DKK2 is epigenetically silenced and inhibits renal cancer progression through apoptotic and cell cycle pathways. Clin Cancer Res 15: 5678-5687, 2009.

8. Hauer K, Calzada-Wack J, Steiger K, Grunewald TG, Baumhoer D Plehm S, Buch T, Prazeres da Costa O, Esposito I, Burdach S and Richter GH: DKK2 mediates osteolysis, invasiveness and metastatic spread in Ewing sarcoma. Cancer Res 73: 967-977, 2013.

9. Matsui A, Yamaguchi T, Maekawa S, Miyazaki C, Takano S, Uetake T, Inoue T, Otaka M, Otsuka H, Sato T, et al: DICKKOPF-4 and -2 genes are upregulated in human colorectal cancer. Cancer Sci 100: 1923-1930, 2009.

10. Roato I, D'Amelio P, Gorassini E, Grimaldi A, Bonello L, Fiori C, Delsedime L, Tizzani A, De Libero A, Isaia G and Ferracini R: Osteoclasts are active in bone forming metastases of prostate cancer patients. PloS One 3: e3627, 2008.

11. Zenzmaier C, Untergasser G, Hermann M, Dirnhofer S, Sampson N and Berger P: Dysregulation of Dkk-3 expression in benign and malignant prostatic tissue. Prostate 68: 540-547, 2008.

12. Kawasaki K, Watanabe M, Sakaguchi M, Ogasawara Y, Ochiai K, Nasu Y, Doihara H, Kashiwakura Y, Huh NH, Kumon H and Date H: REIC/Dkk-3 overexpression downregulates P-glycoprotein in multidrug-resistant MCF7/ADR cells and induces apoptosis in breast cancer. Cancer Gene Ther 16: 65-72, 2009.
13. Yue W, Sun Q, Dacic S, Landreneau RJ, Siegfried JM, Yu J and Zhang L: Downregulation of Dkk3 activates beta-catenin/TCF-4 signaling in lung cancer. Carcinogenesis 29: 84-92, 2008.

14. Zhu J, Zhang S, Gu L and Di W: Epigenetic silencing of DKK2 and Wnt signal pathway components in human ovarian carcinoma. Carcinogenesis 33: 2334-2343, 2012.

15. de la Taille A, Rubin MA, Chen MW, Vacherot F, de Medina SG, Burchardt M, Buttyan R and Chopin D: Beta-catenin-related anomalies in apoptosis-resistant and hormone-refractory prostate cancer cells. Clin Cancer Res 9: 1801-1807, 2003.

16. Liu Z, Rebowe RE, Wang Z, Li Y, Wang Z, DePaolo JS, Guo J, Qian C and Liu W: KIF3a promotes proliferation and invasion via Wnt signaling in advanced prostate cancer. Mol Cancer Res 12: 491-503, 2014.

17. Chen G, Shukeir N, Potti A, Sircar K, Aprikian A, Goltzman D and Rabbani SA: Up-regulation of Wnt-1 and beta-catenin production in patients with advanced metastatic prostate carcinoma: Potential pathogenetic and prognostic implications. Cancer 101: 1345-1356, 2004

18. Verras M and Sun Z: Roles and regulation of Wnt signaling and beta-catenin in prostate cancer. Cancer Lett 237: 22-32, 2006.

19. Lu W, Tinsley HN, Keeton A, Qu Z, Piazza GA and Li Y: Suppression of Wnt/beta-catenin signaling inhibits prostate cancer cell proliferation. Eur J Pharmacol 602: 8-14, 2009.

20. Davies G, Jiang W and Mason M: Cell-cell adhesion molecules and signaling intermediates and their role in the invasive potential of prostate cancer cells. J Urol 163: 985-992, 2000.

21. Whitaker HC, Girling J, Warren AY, Leung H, Mills IG and Neal DE: Alterations in beta.catenin expression and localization in prostate cancer. Prostate 68: 1196-1205, 2008. 